\title{
Incidence of the V600K mutation among melanoma patients with BRAF mutations, and potential therapeutic response to the specific BRAF inhibitor PLX4032
}

\author{
Jill C Rubinstein ${ }^{1}$, Mario Sznol ${ }^{2}$, Anna C Pavlick ${ }^{3}$, Stephan Ariyan ${ }^{4}$, Elaine Cheng ${ }^{5}$, Antonella Bacchiocchi ${ }^{5}$, \\ Harriet M Kluger ${ }^{2}$, Deepak Narayan ${ }^{4}$, Ruth Halaban ${ }^{5^{*}}$
}

\begin{abstract}
Activating mutations in BRAF kinase are common in melanomas. Clinical trials with PLX4032, the mutant-BRAF inhibitor, show promising preliminary results in patients selected for the presence of V600E mutation. However, activating V600K mutation is the other most common mutation, yet patients with this variant are currently excluded from the PLX4032 trials. Here we present evidence that a patient bearing the BRAF V600K mutation responded remarkably to PLX4032, suggesting that clinical trials should include all patients with activating BRAF V600E/K mutations.
\end{abstract}

\section{Commentary}

$\mathrm{BRAF}$ is a serine/threonine protein kinase, encoded on chromosome 7q34, that activates the MAP kinase/ERKsignaling pathway (see KEGG Pathways Database). Approximately $42 \%$ of melanomas harbor activating BRAF mutations (see COSMIC Database, Wellcome Sanger Trust). Most commonly, the valine at amino acid 600 is replaced by glutamate (V600E) through mutation of a single nucleotide (GTG to GAG). Another known mutation at this site involves two nucleotides (GTG to AAG), substituting lysine for valine (V600K) (see Table 1 for statistics on the frequency of these mutations reported in melanoma). The specific BRAF inhibitor PLX4032 (Plexxikon Inc., Berkeley, CA) suppresses the activated oncogenic pathway by inhibiting the ERK kinase cascade. High objective response rates were observed in the phase I clinical trial of PLX4032 in the cohort of melanoma patients selected for tumors with the V600E mutation, and ongoing phase II and phase III clinical trials are limited to those patients with BRAF V600E mutations [1].

The incidence of V600K mutations in melanoma may be greater than previously assumed. In our series of 138

\footnotetext{
* Correspondence: ruth.halaban@yale.edu
${ }^{5}$ Department of Dermatology, Yale University School of Medicine, New

* Correspondence: ruth.halaban@yale.edu
${ }^{5}$ Department of Dermatology, Yale University School of Medicine, New Haven, CT 06520, USA
}

(c) 2010 Rubinstein et al; licensee BioMed Central Ltd. This is an Open Access article distributed under the terms of the Creative

melanomas isolated from patients with disease of varying stage, 42 harbored BRAF mutations (determined by Sanger dideoxy sequencing). Of these, $69 \%$ carried the V600E mutation (15 homozygous), while the remaining $28.6 \%$ carried the V600K variant (8 homozygous), and one carried the V600R (AGG/AGG) mutation. Of note, none of the samples are V600E/V600K heterozygotes, meaning that the $\mathrm{V} 600 \mathrm{~K}$ mutation did not arise from a second alteration at the site of an existing V600E. Altogether, the combined studies show that BRAF V600K mutations are present in $6-30 \%$ of melanoma tumors (Table 1). This broad range cannot be explained by variation in the ratio of primary versus metastatic melanomas or by the different methods used for sequencing (Table 1). Although different methods to detect the mutation were used, most of the studies validated the observations by Sanger dideoxy sequencing. Curiously, the BRAF mutations present in about $84 \%$ of nevi are reported to be of the V600E type [2,3]. Likewise, in our cohort of 14 congenital nevi, we also detected four with BRAF mutations, all heterozygous V600E. Therefore, it is possible that the $\mathrm{V} 600 \mathrm{E}$ and $\mathrm{V} 600 \mathrm{~K}$ mutant melanomas arise from precursor lesions.

PLX4032 is a small molecule inhibitor targeting the activated form of BRAF [4]. In our recent studies on the effects of PLX4032, we demonstrated that the high 
Table 1 Incidence of V600 mutations in melanoma patients

\begin{tabular}{llllll}
\hline $\begin{array}{l}\text { Total V600 } \\
\text { mutants }\end{array}$ & $\begin{array}{l}\text { V600E } \\
\mathbf{( \% )}\end{array}$ & $\begin{array}{l}\text { V600K } \\
(\mathbf{\%})\end{array}$ & $\begin{array}{l}\text { V600 D or } \\
\text { V600R (\%) }\end{array}$ & Reference & Assay \\
\hline 34 & $25(73.5)$ & $4(11.8)$ & $5(14.7)$ & Spittle et al. [7] & $\begin{array}{l}\text { Pyrosequencing, } \\
\text { validated by Sanger dideoxy sequencing }\end{array}$ \\
\hline 10 & $9(90.0)$ & $1(10.0)$ & $0(0.0)$ & Hay et al. [8] & $\begin{array}{l}\text { Melting point analysis, validated by Sanger dideoxy } \\
\text { sequencing }\end{array}$ \\
\hline 44 & $34(77.3)$ & $9(20.5)$ & $1(2.3)$ & Willmore-Payne et al. [9] & $\begin{array}{l}\text { Amplicon melting analysis, validated by Sanger } \\
\text { dideoxy sequencing }\end{array}$ \\
\hline 42 & $29(69)$ & $12(28.6)$ & $1(2.3)$ & $\begin{array}{l}\text { Halaban et al. [5] and Halaban, } \\
\text { unpublished }\end{array}$ & Sanger dideoxy sequencing \\
\hline 50 & $47(94.0)$ & $3(6.0)$ & $0(0.0)$ & Ugurel et al. [10] & Fluorescent capillary SSCP technique \\
\hline 178 & 143 & $29(16.3)$ & $6(3.4)$ & Total Mutations & \\
\hline
\end{tabular}

enzymatic activity of both V600E and V600K BRAF mutants in melanoma cells is suppressed by treatment with PLX4032 [5]. PLX4032 is also known for its paradoxical effect on cells with wild-type BRAF, in which RAF1 is activated (reviewed in [6]). We showed that in BRAF wild-type melanoma cells, PLX4032 stimulated the downstream intracellular signaling pathway, causing cell detachment and motility in metastatic melanoma cells and enhancing cell proliferation in primary melanomas carrying NRAS Q61L mutations. These paradoxical effects highlight the importance of tailoring treatment to the specific genetic composition of the tumor [5].

One of our patients with locally advanced unresectable melanoma involving the skin of the left chest wall, progressing after treatment with topical imiquimod and systemic temozolomide, was referred to another institution for a phase II clinical trial of PLX4032. As part of the eligibility requirements of the trial, paraffin-embedded tissue from a biopsy of a cutaneous lesion was tested and shown to be positive for the BRAF V600E mutation. The patient was treated and achieved an excellent clinical response to PLX4032, associated with substantial reduction in the tumor burden and pain from the lesions. Figure 1 shows the cutaneous lesions before and after treatment with PLX4032. A portion of the tumor was also collected for laboratory research studies according to a protocol approved by Yale University Human Investigations Committee. Written informed consent was obtained from the patient for publication of this case report and accompanying images. A copy of the written consent is available for review by the Editorin-Chief of this journal. Upon retesting for BRAF

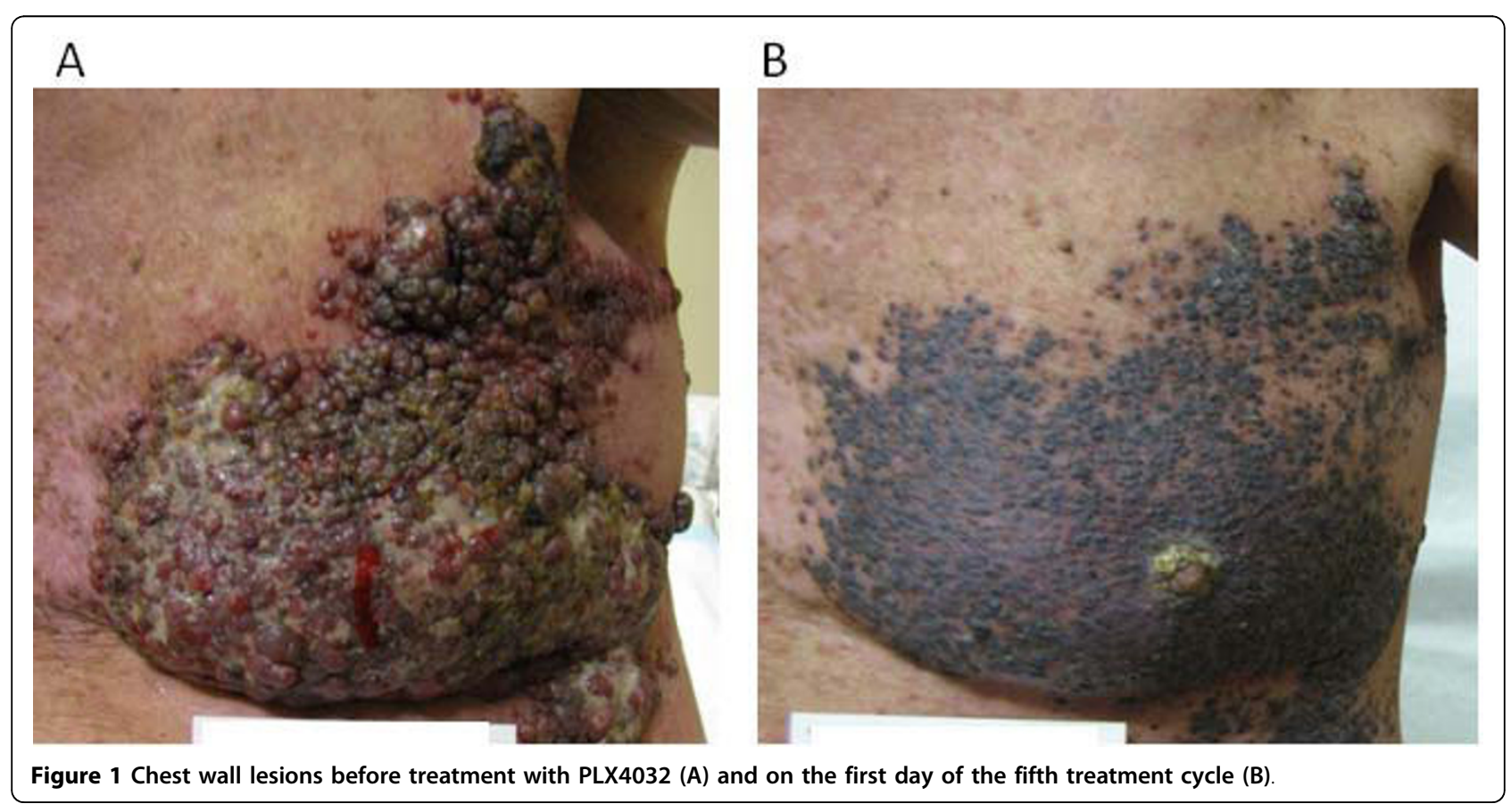




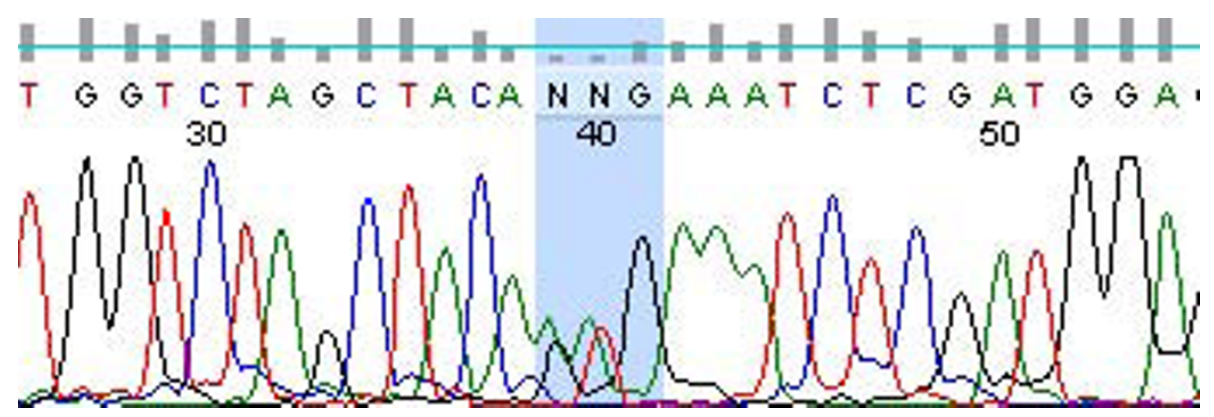

Figure 2 Electropherogram from Sanger dideoxy sequencing showing the patient's melanoma tumor BRAF codon 600 mutation (AAG/GTG), encoding V600K/WT protein.

mutation by Sanger dideoxy sequencing, it was shown that in fact he carried the V600K allele (Figure 2, assay repeated for validation).

\section{Conclusions}

Our data, and those of others reported in the literature, indicate that the incidence of BRAF V600K mutations in melanoma patients appears to be higher than is commonly assumed. It can be present in up to $30 \%$ of patients bearing BRAF V600 mutations, potentially representing up to $10 \%$ of all melanoma patients. Patients with BRAF V600K mutations are currently excluded from clinical trials with PLX4032, although the assay methodology used for the trial may not discriminate between the V600E and V600K mutations. Our preclinical data demonstrating similar kinase activity of the V600K and V600E mutations, together with clear evidence of clinical activity of PLX4032 in a patient with a documented V600K mutation, suggest that melanoma patients with V600K mutations should be included in current and future trials of BRAF inhibitors.

\section{Acknowledgements}

This work was supported by the Yale SPORE in Skin Cancer funded by the National Cancer Institute grant number 1 P50 CA121974 (R. Halaban, PI). Written consent for publication was obtained from the patient.

\section{Author details}

'Department of Pathology, Yale University School of Medicine, New Haven, CT 06520, USA. ${ }^{2}$ Section of Medical Oncology, Yale University School of Medicine, New Haven, CT 06520, USA. 'epartment of Medical Oncology, New York University, New York City, New York 10016, USA. ${ }^{\text {PPlastic and }}$ Reconstructive Surgery, Yale University School of Medicine, New Haven, CT 06520, USA. ${ }^{5}$ Department of Dermatology, Yale University School of Medicine, New Haven, CT 06520, USA.

\section{Authors' contributions}

$J \mathrm{R}$, compiled literature search and participated in writing the manuscript; MS conceived the report and participated in writing the manuscript; ACP, treated the patient with PLX4032; SA, excised tumors and provided the material for research; $\mathrm{EC}$, performed the BRAF mutation analysis; $\mathrm{AB}$, collected tumor material and established melanoma cells in culture; HMK, participated in writing of the manuscript; DN, excised tumors and provided the material for research; $\mathrm{RH}$, in charge of analyzing tumor specimens and participated in writing the manuscript. All authors have read and approved the final manuscript.

\section{Competing interests}

The authors declare that they have no competing interests.

Received: 12 May 2010 Accepted: 14 July 2010 Published: 14 July 2010

\section{References}

1. Flaherty K, Puzanov I, Sosman J, Kim K, Ribas A, McArthur G, Lee RJ, Grippo JF, Nolop K, Chapman P: Phase I study of PLX4032: Proof of concept for V600E BRAF mutation as a therapeutic target in human cancer. J Clin Oncol (Meeting Abstracts) 2009, 27:9000.

2. Uribe P, Wistuba I, González S: BRAF mutation: a frequent event in benign, atypical, and malignant melanocytic lesions of the skin. Am J Dermatopathol 2003, 25:365-370.

3. Poynter J, Elder J, Fullen D, Nair R, Soengas M, Johnson T, Redman B, Thomas N, Gruber S: BRAF and NRAS mutations in melanoma and melanocytic nevi. Melanoma Res 2006, 16:267-273.

4. Sala E, Mologni L, Truffa S, Gaetano C, Bollag G, Gambacorti-Passerini C: BRAF silencing by short hairpin RNA or chemical blockade by PLX4032 leads to different responses in melanoma and thyroid carcinoma cells. Mol Cancer Res 2008, 6:751-759.

5. Halaban R, Zhang W, Bacchiocchi A, Cheng E, Parisi F, Ariyan S, Krauthammer M, McCusker J, Kluger Y, Sznol M: PLX4032, a selective BRAF (V600E) kinase inhibitor, activates the ERK pathway and enhances cell migration and proliferation of BRAF melanoma cells. Pigment Cell Melanoma Res 2010, 23:190-200.

6. Pratilas C, Solit D: Targeting the MAPK Pathway: Physiological Feedback and Drug Response. Clin Cancer Res 2010, 16:3329-34.

7. Spittle C, Ward M, Nathanson K, Gimotty P, Rappaport E, Brose M, Medina A, Letrero R, Herlyn M, Edwards R: Application of a BRAF pyrosequencing assay for mutation detection and copy number analysis in malignant melanoma. J Mol Diagn 2007, 9:464-471.

8. Hay R, MacRae E, Barber D, Khalil M, Demetrick D: BRAF mutations in melanocytic lesions and papillary thyroid carcinoma samples identified using melting curve analysis of polymerase chain reaction products. Arch Pathol Lab Med 2007, 131:1361-1367.

9. Willmore-Payne C, Holden J, Tripp S, Layfield L: Human malignant melanoma: detection of BRAF- and c-kit-activating mutations by highresolution amplicon melting analysis. Hum Pathol 2005, 36:486-493.

10. Ugurel S, Thirumaran R, Bloethner S, Gast A, Sucker A, Mueller-Berghaus J, Rittgen W, Hemminki K, Becker J, Kumar R, Schadendorf D: B-RAF and NRAS mutations are preserved during short time in vitro propagation and differentially impact prognosis. PLoS One 2007, 2:e236.

doi:10.1186/1479-5876-8-67

Cite this article as: Rubinstein et al:: Incidence of the V600K mutation among melanoma patients with BRAF mutations, and potential therapeutic response to the specific BRAF inhibitor PLX4032. Journal of Translational Medicine 2010 8:67. 